\title{
Synthesis and Characterization of Ceria Supported Platinum via Facile Wet Impregnation from Cerium Based Precursors
}

\author{
Alam BB' ${ }^{1}$, O'Toole AW$^{2}$, Matyi RJ', Brenner SA ${ }^{1^{*}}$ \\ ${ }^{1}$ Colleges of Nanoscale Science and Engineering, SUNY Polytechnic Institute, Albany, New York, USA \\ ${ }^{2}$ ThermoAura Inc, Colonie, New York, USA
}

\begin{abstract}
Platinum $(\mathrm{Pt})$ based catalysts are used in fuel cell applications for the high efficiency, low temperature and non-pollutant producing method of gas conversion, however the drawbacks to commercialization include the high cost, durability and reliability of the process. Ceria supported Pt catalyst is a more promising candidate in the low temperature water-gas shift reaction because such a supported catalyst not only reduces the cost and temperature, but also achieves a greater extent of reduction and promotes the catalytic functions of Pt. A facile wet impregnation method using chloroplatinic acid in combination with cerium based precursors, such as ceria from a sol-gel peroxo method and cerium (III) acetate hydrate, have been used to synthesize ceria supported Pt. Support characteristics such as size and agglomeration state vary according to the type of precursor used and affect Pt coverage on the support. In addition, the method using cerium (III) acetate does not require an initial thermal conversion step to ceria $\mathrm{Pt}$ coated the ceria independent of the crystallite size and agglomeration state of the particles, but the Pt coverage varied depending on the precursor used.
\end{abstract}

Keywords: Catalyst; Platinum; Ceria; Nanoparticles; Impregnation

\section{Introduction}

The synthesis of suitable support materials for platinum $(\mathrm{Pt})$ catalysis have been under investigation as expensive platinum based catalysts are used in fuel cell applications for the high efficiency, low temperature and non-pollutant producing method of gas conversion. While these advantages make Pt a promising material for catalysis, the high cost, durability and reliability limit commercialization [1]. To overcome these challenges and to make the catalyst work more effectively the surface area of the catalyst needs to be maximized. This is where a support may come into play, and the support may either be inert or participate in the reaction. Pt catalysts are widely used in the water-gas shift (WGS) reaction, where carbon monoxide and water vapor react to form carbon dioxide and hydrogen known as the water gas. This has the undesirable component of carbon monoxide that needs to be removed, requiring different reaction conditions and high temperatures. When a support such as a metal oxide or carbon is added to a catalyst, there has been evidence of the migration of oxide species into the metal and additional interaction, called the Strong Metal Support Interaction (SMSI). This interaction, even though initially regarded as having a negative impact on the reaction rates of certain reactions such as alkane hydrogenolysis, is known to have a positive impact on the $\mathrm{CO}-\mathrm{H}_{2}$ reaction [2]. Supports are highly desirable for certain reactions as they not only increase the effective surface area of the catalyst, but also decrease the temperature substantially for the conversion reaction.

While several materials such as $\mathrm{C}$, Sn-C have already been investigated for this purpose [3], ceria supported Pt catalyst is a more promising candidate in the low temperature WGS reaction because it has not only been known to reduce the temperature, but also achieve a greater extent of reduction [4]. This is due to the fact that $\mathrm{CO}$ chemisorbs on to the metal $(\mathrm{Pt})$ surface and then is subsequently oxidized by the support via interaction of the surface $\mathrm{OH}$ groups available on the ceria surface. A higher reduced (metal coordination) surface available leads to a greater number of germinal $\mathrm{OH}$ groups on the ceria surface that react with $\mathrm{CO}$ to produce formates. In addition, the Pt metal available on the surface promotes the reduction of ceria, and $\mathrm{Pt} /$ ceria catalysts are an order of magnitude higher in activity compared to $\mathrm{Cu}, \mathrm{Co}, \mathrm{Ni}$ and Fe ceria catalysts at lower temperatures [5].

Ceria exists in both the +3 and the +4 oxidation states and can switch between the two oxidation states relatively easily [6]. Ceria acts as a suitable support because the oxide ion vacancies are formed relatively easily, which play a vital role in catalysis, while the metal ion $(\mathrm{Pt})$ acts as the CO adsorption site. In this case, the oxygen from the feed stream not only gets oxidized but there are surface reactions of the $\mathrm{CO}$ on the support with the oxide species of the support. Also, addition of Pt leads to a decrease of oxygen occupancy and creation of defects. The $\mathrm{Ce}_{1-\mathrm{x}} \mathrm{Pt}_{\mathrm{x}} \mathrm{O}_{2}$ system reaches equilibrium after the evolution of lattice oxygen, and even a large decrease in $\mathrm{CO}$ is observed without the stoichiometric formation of $\mathrm{H}_{2}$ despite the continuous supply of $\mathrm{H}_{2} \mathrm{O}$, which indicates the use of lattice oxygen at least in the initial stages. The addition of $\mathrm{CO}$ provides a reducing environment and the eventual restoration of the catalyst can occur through the dissociation of the $\mathrm{H}_{2} \mathrm{O}$ molecules [7]. A bulk, defect free ceria support is unfavorable for this type of reaction to take place. However, the number of these defect sites changes upon thermal treatment. Thermal treatment also activates the reverse oxygen spillover from the support with the presence of coadsorbed oxygen near the $\mathrm{CO}$ sites and electronic metal-support interactions by the $\mathrm{CO}$ sites [8].

In some instances, the shape of the ceria support affects the rate of the WGS reaction where smaller sized particles are better as supports and nanorod shaped support are more effective than nanocube shaped supports [9]. In addition, with decreasing particle size and

*Corresponding author: Brenner SA, Colleges of Nanoscale Science and Engineering, SUNY Polytechnic Institute, Albany, New York, USA, Tel: 518-9567224; E-mail: sbrenner@sunycnse.com

\section{Received April 29, 2015; Accepted May 27, 2015; Published June 03, 2015}

Citation: Alam BB, O'Toole AW, Matyi RJ, Brenner SA, et al. (2015) Synthesis and Characterization of Ceria Supported Platinum via Facile Wet Impregnation from Cerium Based Precursors. J Adv Chem Eng 5: 123. doi:10.4172/20904568.1000123

Copyright: ( 2015 Alam BB et al. This is an open-access article distributed under the terms of the Creative Commons Attribution License, which permits unrestricted use, distribution, and reproduction in any medium, provided the original author and source are credited. 
coordination, metals with fewer neighbors are able to bind to adsorbates more strongly. This behavior is enhanced with an appropriate support, such as ceria, where special sites at the interface of the metal and support either influence the properties of the adsorbate or lattice size adjustment of metal, creating a strain that influences the strength of the metal-adsorbate bonding. The probability of adsorption of water on the surface is very low with only the metal catalyst; with ceria, the WGS reaction has shown to be 20 folds higher, while embedding Pt particles in ceria fibers often enhances this activity to 100 -fold higher. This is due to the fact that Pt particles experience large electronic perturbations in contact with ceria, which enhances the ability to dissociate the $\mathrm{O}-\mathrm{H}$ bonds [10].

The enhancing capability of ceria for noble metal catalysis has profound impacts on cost reduction, and methods are still being investigated to reduce the amount of noble metal and maximize the support interaction. This study investigates the synthesis, characterization and comparison of the structural characteristics of both bare Pt nanoparticles and ceria supported Pt on nanosized ceria particles and high uniform coating of Pt on the support using facile wet impregnation methods from two cerium based precursors. Using different cerium based precursors results in various size ranges and particle characteristics and coverage of $\mathrm{Pt}$ on the support.

\section{Materials and Methods}

Three methods were used to synthesize three materials for this investigation: 1) Reduction of a Pt precursor to produce bare $\mathrm{Pt}$ nanoparticles. Two wet impregnation methods for ceria supported $\mathrm{Pt}$ with Pt nanoparticles coated on ceria from 2) already synthesized ceria nanoparticles 3) Using cerium acetate as a precursor.

\section{Synthesis of Pt nanoparticles}

Pt nanoparticles were produced by the ethylene glycol assisted sodium borohydride $\left(\mathrm{NaBH}_{4}\right)$ reduction of the Pt precursor. The complex that forms by this reaction serves two purposes: a reducing agent and a stabilizer for to limit the Pt particle growth and keep narrow size ranges. This is desirable because all of the steps can be carried out at room temperature [11]. $339 \mathrm{mg}$ of chloroplatinic acid hexahydrate (Alfa Aesar, USA) was dispersed in $100 \mathrm{~mL}$ of ethylene glycol (99\%, Alfa Aesar, USA). In a separate beaker, $15.25 \mathrm{~g}$ of sodium borohydride (Sigma Aldrich, USA) was added to $200 \mathrm{~mL}$ of ethylene glycol. The previous chloroplatinic acid hexahydrate and ethylene glycol mixture complex was added under vigorous stirring with a magnetic stirrer. Bubbling and immediate color change from orange to black was observed. This mixture was stirred for 2 hours and the particles were allowed to settle overnight. The excess solution was then decanted off, and the particles were centrifuged, washed three times with DI water and dried in an oven at $70^{\circ} \mathrm{C}$ overnight (Figure 1).

\section{Synthesis of ceria supported Pt from sol-gel ceria nanoparticles by wet impregnation}

$1.79 \mathrm{~g}$ of ceria particles produced from a facile sol-gel peroxo method of synthesis and annealed in open atmosphere, $2.62 \mathrm{~g}$ of chloroplatinic acid hexahydrate were dispersed in $200 \mathrm{~mL}$ of ethanol (Sigma Aldrich, USA) and thoroughly stirred overnight with a magnetic stirrer. The temperature of the stir plate was then increased from $80^{\circ} \mathrm{C}$ to $120^{\circ} \mathrm{C}$ and allowed to evaporate over 5 hours until the volume of the dispersion was $50 \mathrm{~mL}$. This was then dried in an oven at $80^{\circ} \mathrm{C}$ overnight. The particles were then annealed in forming gas $\left(5 \% \mathrm{H}_{2} / 95 \% \mathrm{Ar}\right.$, Air Liquide, USA) at $525^{\circ} \mathrm{C}$ for 4 hours (Figure 2).

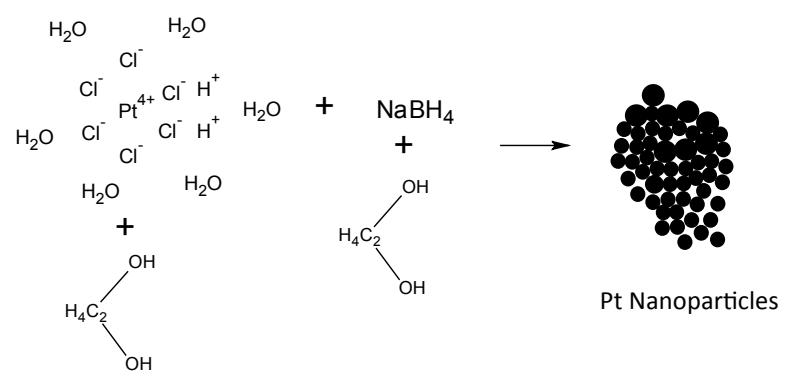

Figure 1: Schematic of Pt nanoparticle synthesis from ethylene glycol assisted sodium borohydride reduction of chloroplatinic acid hexahydrate.

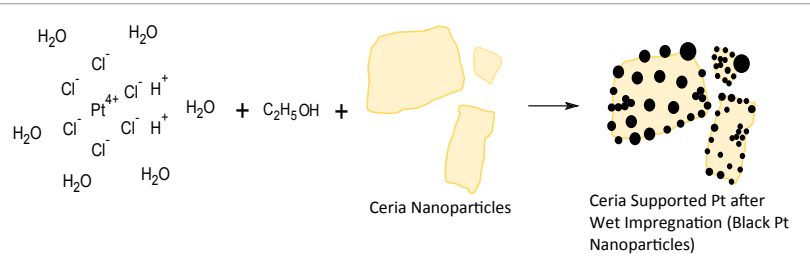

Figure 2: Schematic of wet impregnation method for ceria supported Pt.

\section{Synthesis of ceria supported Pt from cerium (III) acetate by wet impregnation}

$2.03 \mathrm{~g}$ of cerium (III) acetate hydrate (Sigma Aldrich, USA) was dispersed in $200 \mathrm{~mL}$ of ethanol and $2.70 \mathrm{~g}$ of chloroplatinic acid hexahydrate and stirred overnight. The mixture was then allowed to evaporate until $50 \mathrm{~mL}$ and then dried in an oven overnight at $80{ }^{\circ} \mathrm{C}$. This was then annealed in forming gas for 4 hours at $525^{\circ} \mathrm{C}$.

\section{Analysis methods}

For Scanning Electron Microscopy (SEM), powders were dispersed on double sided carbon tape on Si wafer scraps and mounted on SEM stubs. A LEO 1550 Gemini Schottky Field Emission Gun (Zeiss, USA) equipped with Energy Dispersive X-Ray Spectroscopy (EDS) was used to image the surface appearance of the Pt on $\mathrm{CeOx}$ powders.

Approximately $5 \mathrm{mg}$ of each synthesized particle sample were dispersed in $50 \mathrm{~mL}$ DI water and ultrasonicated for Transmission Electron Microscopy (TEM) analysis. For TEM, approximately $3 \mu \mathrm{l}$ of the dispersion was pipetted onto a holey carbon grid (400 mesh, Ted Pella, USA) and air-dried overnight.

A JEOL 2010 Field Emission Gun (JEOL, Japan) operating at $200 \mathrm{kV}$ was used to image the samples on holey carbon grids. TEM bright field mode was used to image both the Pt and the Pt coated ceria particles for size and morphological investigation while selected area electron diffraction was used to determine the crystal structure of the particles. TEM diffraction patterns were obtained for the bare Pt and the ceria supported Pt.

For X-Ray Diffraction (XRD) analysis, these dispersions were deposited on glass slides and allowed to dry. Analyses were performed on a Bruker D8 Discover High Resolution X-ray diffractometer (Bruker, Germany) using $\mathrm{Cu} \mathrm{K}_{\alpha}$ radiation from a sealed tube $(40 \mathrm{kV}, 40$ $\mathrm{mA}) \cdot \theta-2 \theta$ scans were recorded from $20^{\circ}$ to $85^{\circ}(2 \theta)$, with step size $0.02^{\circ}$ and count time 10 seconds/point (approximately 9 hours per scan). These samples were nanopowders and were analyzed using a powder diffraction method called Rietveld refinement to overcome issues associated with powder samples analysis such as peak broadening and 
large background. The Rietveld method refines user-selected parameters to minimize the difference between an experimental pattern (observed data) and a model based on the hypothesized crystal structure and instrumental parameters (calculated pattern). A National Institute of Standards and Technology (NIST) Standard Reference Material (SRM) 674b (ceria) was used as the standard for obtaining instrument parameters. The fit was obtained using NIST SRM certificate values of lattice parameter (5.411651 $\AA$ ), crystal size ( $380.6 \mathrm{~nm}$, no microstrain), assuming ideal stoichiometry (100\% site occupancy), and no preferred orientation. This fit was used to determine the instrument function parameters, which were also employed in subsequent fits. A non-linear least squares fitting was performed to minimize the value of " $R$-weighted pattern" $\left(R_{\mathrm{wP}}\right)$. The peaks in the diffraction profile were indexed using the appropriate JCPDS card number for Pt and ceria (Figure 3 ). The calculated and the measured pattern show good agreement. XRD data for the NIST ceria standard showed that the ceria particles existed in the cubic fluorite structure. TEM Electron diffraction patterns also confirmed the existence of the particles in the cubic fluorite structure.

\section{Results}

Ceria supported Pt particles were synthesized from two wet impregnation methods using two cerium-based precursors: ceria nanoparticles from a sol-gel peroxo method and cerium (III) acetate hydrate. Standalone bare $\mathrm{Pt}$ nanoparticles were created for crystal structural comparison purposes. The resulting physical appearance of the samples was a black powder. Electron diffraction patterns obtained showed that both ceria supported Pt and Pt nanoparticles crystalized in the cubic fluorite phase with the presence of the $111,220,311$ peaks in the diffraction pattern that was indexed.

SEM images of particles showed that Pt particles coated the support. EDS confirmed the presence of both $\mathrm{Pt}$ and Ce on the particles (Figure 4). TEM provides a better visualization of the particles and the support due to higher resolution and improved contrast mechanisms.

In TEM, the metal particles (Pt) appear darker whereas oxides appear lighter, and the contrast provides convenient visualization of the Pt coating on the ceria particles. TEM images of Pt particles synthesized by this method show that the Pt is less than $10 \mathrm{~nm}$ in size (Figure 3). Even though previous authors had reported that ethylene

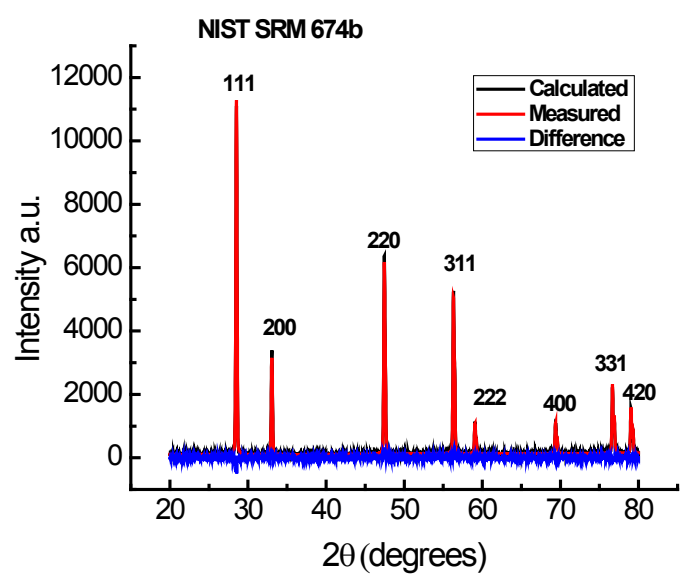

Figure 3: Diffraction profile for the NIST SRM 674b (ceria) powder which showed good agreement between the calculated and the measured pattern. This pattern was used to determine instrument parameters employed in the subsequent fits and also demonstrated the cubic fluorite structure of the ceria crystals. glycol assisted sodium borohydride reduction reduce agglomeration of the resulting Pt particles that are produced from this method, TEM images of Pt particles synthesized by this method showed agglomerated particles. However, using wet impregnation methods, the Pt particles formed clusters around the ceria support coating the particles and did not agglomerate among themselves. Isolated Pt particles were not found, and all Pt particles primarily stuck to the ceria crystallites. Therefore, using a support not only prevents the Pt nanoparticles from agglomerating but also increases the effective surface area of the $\mathrm{Pt}$ nanoparticles. Rietveld XRD diffraction profiles also revealed broader peaks for the bare Pt particles and Pt supported ceria from acetate precursor, showing that the particles synthesized were nanoparticle range in size. A sharper diffraction profile for the Pt supported ceria from the sol-gel method showed that the particle sizes from the sol-gel were larger and resembled more bulk size particles.

XRD data showed the average size of the crystallite to be close to values obtained from TEM for single or agglomerated particles. This method produces Pt that consistently coats the ceria. Smaller ceria particles tended to have a higher proportion of area coated by Pt.

\section{Crystal structure characteristics of $\mathrm{Pt}$ and ceria supported $\mathrm{Pt}$}

XRD data showed the existence of a two phase mixture of Pt and ceria for the supported particles, with $68.70 \%$ ceria and $31.30 \% \mathrm{Pt}$ for the sol-gel ceria particles and $78.88 \%$ ceria and $21.12 \%$ Pt for the acetate based ceria particles (Figure 5). XRD data using the Rietveld refinement confirmed that the particles existed in the cubic fluorite structure with the indexing of allowed reflections in the diffraction profile. The reported lattice parameter for Pt was close to the $3.92 \AA$ reported in the literature [12], while the lattice paramaters for the ceria particles were close to the value of $5.41 \AA$ from the NIST SRM $674 \mathrm{~b}$. The lattice parameters for the smaller ceria acetate particles were larger than the bulk value (NIST SRM 674b) due to increased lattice strain in the smaller particles [13]. The lattice parameters of the Pt nanoparticles were similar with or without the ceria support. Crystallite sizes for the smaller ceria particles from acetate showed the same crystallite sizes with and without $\mathrm{Pt}$, while the crystallite sizes for the ceria with the sol-gel method were slightly different owing to the large variation in crystallite sizes from the sol-gel method but were consistent with the sizes observed from TEM imaging. The Pt crystallite sizes were measured to be larger on the support than the bare synthesized particles. However, TEM images showed that the particles were similar in size to the bare sized particles on the support whereas XRD measurements revealed a greater crystallite size, which suggests a more continuous coating of Pt on the support (Table 1).

\section{Conclusion}

The processes used in this study produced Pt particles that consistently coated ceria supports. Both ceria and Pt particles are polycrystalline with the cubic fluorite structure but varying lattice parameters due differences in interplanar spacing forming a two phase mixture. The results from XRD also demonstrated that there was a variation in coverage of Pt on the support with the larger solgel particles having $31.30 \% \mathrm{Pt}$ coverage and the smaller acetate based ceria particles consisting of $21.12 \%$ Pt coverage. However, in both cases, Pt covered ceria despite the size and degree of agglomeration, and in some cases, even continuously coated the nanoparticles. The acetate method of wet impregnation did not require initial conversion to ceria [14] and reduces one thermal step in processing which would be an advantageous cost reduction strategy when such a support based catalyst is heavily commercialized. 
Citation: Alam BB, O'Toole AW, Matyi RJ, Brenner SA et al (2015) Synthesis and Characterization of Ceria Supported Platinum via Facile Wet Impregnation from Cerium Based Precursors. J Adv Chem Eng 5: 123. doi:10.4172/2090-4568.1000123

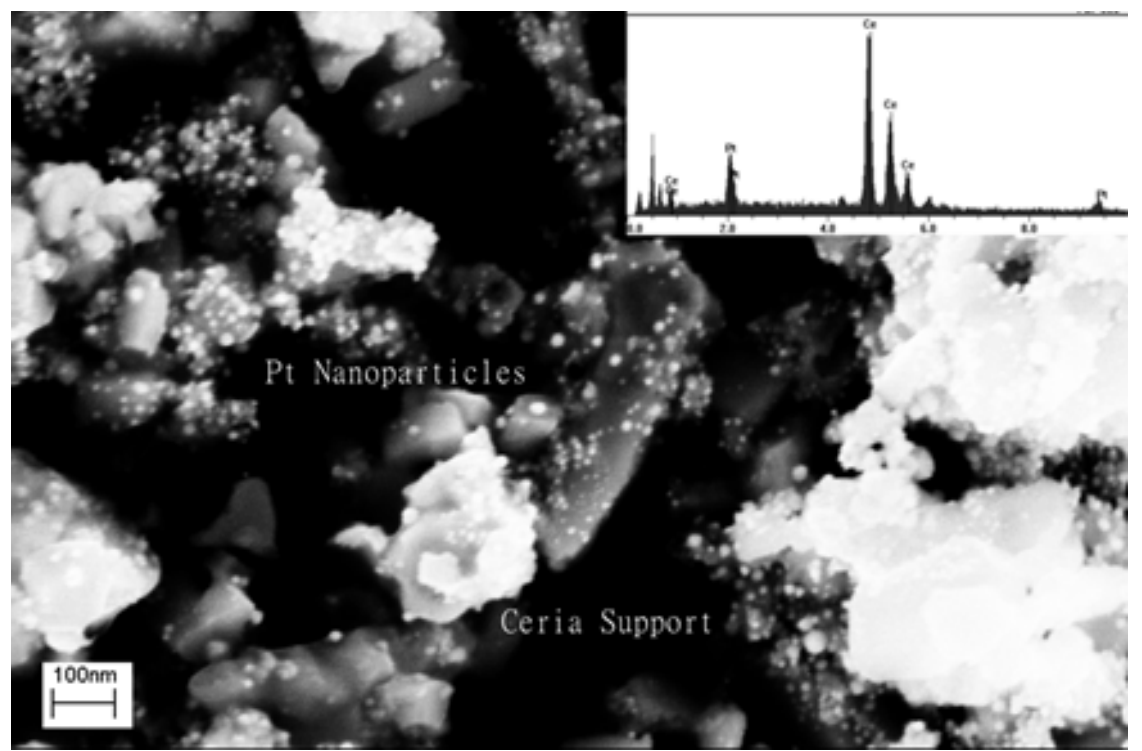

Figure 4: SEM image of ceria supported Pt nanoparticles. Pt nanoparticles (smaller white specks) attach to the surface of the ceria support (larger supporting crystallites). Inset is EDS spectrum of a ceria supported Pt crystallite showing the elemental presence of Ce and Pt on these particle clusters.
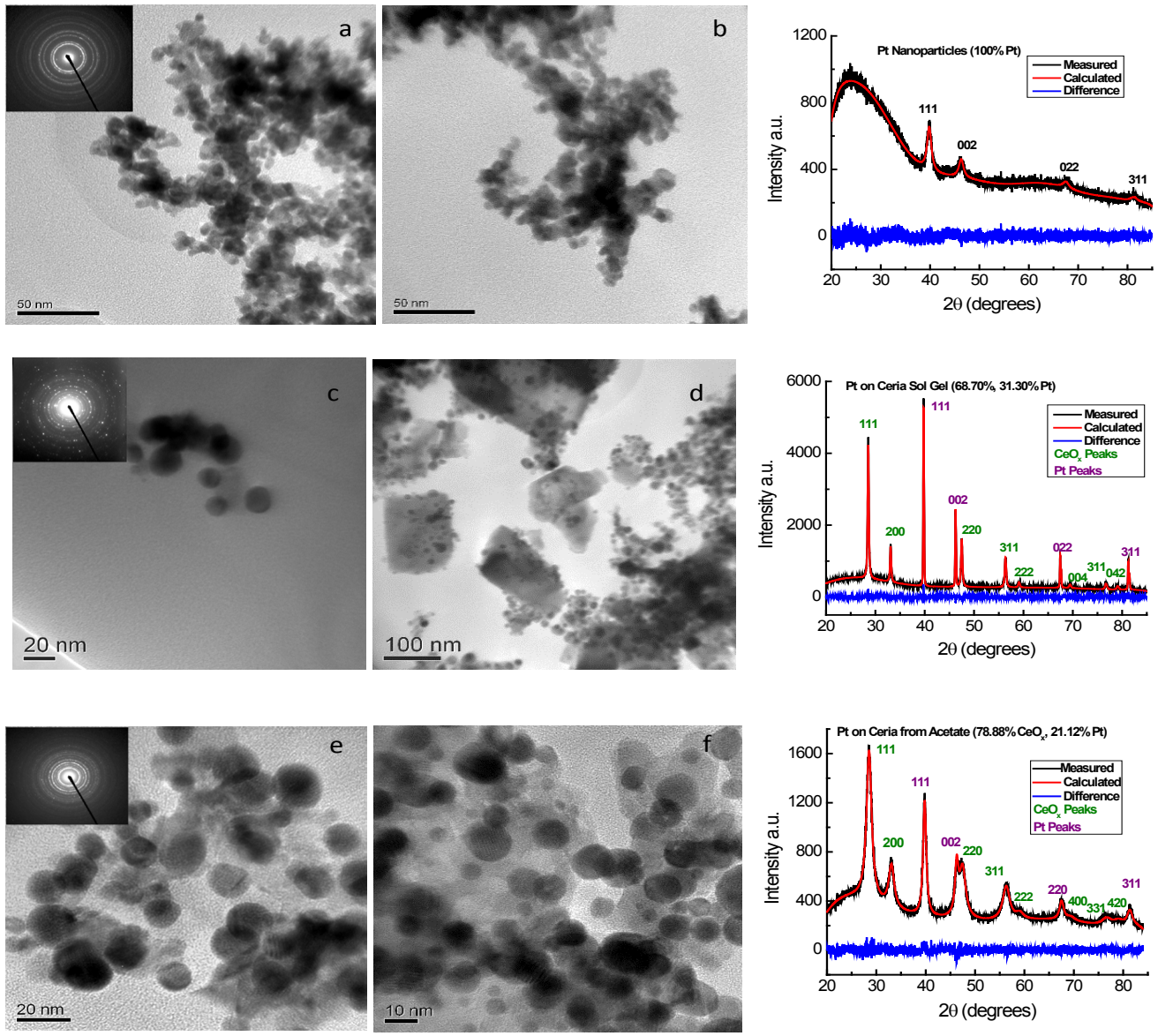

Figure 5: TEM images of Pt particles with insets of selected area electron diffraction patterns. Images a and b show standalone bare Pt nanoparticles. Images $c$ and d show the ceria supported Pt from the sol-gel peroxo wet impregnation methods. To the right of each set of images are the Rietveld refined powder diffraction profile. All patterns contained the allowed fcc reflections after indexing, with Pt exhibiting the 111, 002, 220 and 311 reflections and ceria exhibiting the 111, 200, 220, $311,222,400,331$ and 420 . The peaks for the same reflections in $\mathrm{Pt}$ and ceria are at different angles due the different interplanar spacings in the Pt and the ceria. 
Citation: Alam BB, O'Toole AW, Matyi RJ, Brenner SA et al (2015) Synthesis and Characterization of Ceria Supported Platinum via Facile Wet Impregnation from Cerium Based Precursors. J Adv Chem Eng 5: 123. doi:10.4172/2090-4568.1000123

Page 5 of 5

\begin{tabular}{|c|c|c|c|c|c|}
\hline Sample & $\mathrm{CeO}_{2} \mathrm{a}_{\mathrm{o}}(\AA)$ & $\mathrm{CeO}_{2} \mathrm{~L}(\mathrm{~nm})$ & $P t a_{o}(\AA)$ & Pt L (nm) & $\mathbf{R}_{\mathrm{wp}}$ \\
\hline Nanoceria (Ce acetate) & $5.4233 \pm 0.0019$ & $7.1 \pm 0.1$ & & & 5.641 \\
\hline Nanoceria (sol-gel) & $5.4119 \pm 0.0007$ & $70.2 \pm 0.5$ & & & 6.005 \\
\hline Pt with ceria from sol-gel & $5.4127 \pm 0.0002$ & $48.8 \pm 0.5$ & $3.9234 \pm 0.0001$ & $246.3 \pm 6.9$ & 5.927 \\
\hline Pt with ceria from acetate & $5.4170 \pm 0.0011$ & $7.0 \pm 0.1$ & $3.9223 \pm 0.0006$ & $13.0 \pm 0.2$ & 5.492 \\
\hline $\mathrm{Pt}$ (no ceria) & & & $3.9268 \pm 0.0041$ & $9.3 \pm 0.4$ & 5.017 \\
\hline NIST SRM 674b & 5.411651 & 380.6 & & & 7.113 \\
\hline
\end{tabular}

Table 1: X-Ray Diffraction data for synthesized Pt and ceria supported $P t$, where $a_{0}$ is the lattice parameter and $L$ the crystallite size.

\section{Acknowledgment}

The authors would like to acknowledge all members of CNSE metrology for their training and assistance on tools and the NanoHealth \& Safety Center (New York) for funding support.

\section{References}

1. Lim DH, Lee WD, Choi DH, Lee HI (2010) Effect of ceria nanoparticles into the $\mathrm{Pt} / \mathrm{C}$ catalyst as cathode material on the electrocatalytic activity and durability for low-temperature fuel cell. Appl Catal B 94: 85-96.

2. Bowker M, Stone P, Morrall P, Smith R, Bennett R, et al. (2005) Model catalyst studies of the strong metal-support interaction: Surface structure identified by STM on Pd nanoparticles on TiO2(110). J Catal 234: 172-181.

3. Lopez-Suarez FE, Bueno-Lopez A, Eguiluz KIB, Salazar-Banda GR (2014) Pt$\mathrm{Sn} / \mathrm{C}$ catalysts prepared by sodium borohydride reduction for alcohol oxidation in fuel cells: Effect of the precursor addition order. J Power Sources 268: 225232.

4. Buitrago R, Ruiz-Martínez J, Silvestre-Albero J, Sepúlveda-Escribano A Rodríguez-Reinoso F (2012) Water gas shift reaction on carbon-supported $\mathrm{Pt}$ catalysts promoted by CeO2. Catal Today 180: 19-24.

5. Jacobs G, Chenu E, Patterson PM, Williams L, Sparks D, et al. (2004) Watergas shift: Comparative screening of metal promoters for metal/ceria systems and role of the metal. Appl Catal A Gen 258: 203-214.

6. Lin M, Fu ZY, Tan HR, Tan JPY, Ng SC, et al. (2012) Hydrothermal synthesis of $\mathrm{CeO} 2$ nanocrystals: Ostwald ripening or oriented attachment? Cryst Growth Des 12: 3296-3303.
7. Deshpande PA, Hegde MS, Madras G (2010) Pd and Pt ions as highly active sites for the water-gas shift reaction over combustion synthesized zirconia and zirconia-modified ceria. Appl Catal B: Environmental 96: 83-93.

8. Happel M, Myslivecek J, Johanek V, Dvorak F, Stetsovych O, et al. (2012) Adsorption sites, metal-support interactions, and oxygen spillover identified by vibrational spectroscopy of adsorbed $\mathrm{CO}$ : A model study on Pt/ceria catalysts. J Catal 289: 118-126.

9. Ciftci A, Ligthart DAJ, Pastorino P, Hensen EJM (2013) Nanostructured ceria supported $\mathrm{Pt}$ and $\mathrm{Au}$ catalysts for the reactions of ethanol and formic acid. App Catal B: Environmental 130-131: 325-335.

10. Campbell CT (2012) Catalyst-support interactions: Electronic perturbations Nat Chem 4: 597-598.

11. Kim P, Joo JB, Kim W, Kim J, Song IK, et al. (2006) NaBH4-assisted ethylene glycol reduction for preparation of carbon-supported $\mathrm{Pt}$ catalyst for methanol electro-oxidation. J Power Sources 160: 987-990.

12. Yu P, Pemberton M, Plasse $P$ (2005) $P t C o / C$ cathode catalyst for improved durability in PEMFCs. J Power Sources 144: 11-20.

13. Zhang F, Jin Q, Chan S (2004) Ceria nanoparticles: Size, size distribution, and shape. J Appl Phys 95: 4319-4326.

14. Damyanova S, Bueno JMC (2003) Effect of $\mathrm{CeO} 2$ loading on the surface and catalytic behaviors of $\mathrm{CeO} 2-\mathrm{Al} 2 \mathrm{O} 3-$ supported Pt catalysts. Appl Catal A Gen 253: 135-150. 